\title{
The promise, perceptions, and pitfalls of immunoassays for autoantibody testing in myositis
}

Sarah L. Tansley ${ }^{1 *} \mathbb{D}$, Julia Snowball1, John D. Pauling ${ }^{1}$, Anya Lissina ${ }^{1}$, Masataka Kuwana², Lisa G. Rider Johan Rönnelid ${ }^{4}$, Neil J. McHugh ${ }^{1}$ and on behalf of the International Myositis Assessment and Clinical Studies (IMACS) Group Myositis Autoantibody Scientific Interest Group

\begin{abstract}
Background: A myositis-specific autoantibody can now be identified in the majority of patients with myositis. They identify homogeneous patient subgroups and are key tools in developing a personalized approach to disease management. There is substantial clinical interest in exploiting myositis autoantibodies as biomarkers, and consequently, a large number of commercial assays have been developed for their detection. These assays are already in widespread clinical use. In order to better understand perceived concerns from the international myositis community in relation to the reliability of these assays and how they are being used, we conducted a survey of international myositis experts, all of whom were members of the International Myositis Assessment and Clinical Studies group.

Results: We collected data on the types of assay used, manufacturers, and the nature of the report provided by different laboratories and received 111 complete responses. Respondents also provided information on how they used the different assays, their confidence in the results, and how this influenced their clinical practice. Enzyme immunoassay/ELISA was the most popular assay method used worldwide followed by line blot. Line blot was the most popular method used in Europe. Despite concerns from over $80 \%$ of respondents regarding false-positive and false-negative results with the assay used by their laboratory, over $80 \%$ reported that the identification of a myositis autoantibody influenced their diagnostic confidence, the information they provided to a patient, and their recommended treatment.

Conclusions: In spite of ongoing concerns from the majority of users regarding the reliability of the results, myositis-specific autoantibody testing, using commercial immunoassays, is being used globally to inform clinical decision-making. These findings highlight the need for urgent guidance on the use of myositis autoantibody testing and on the interpretation of results. Knowledge of the reliability of currently available assays is essential given the importance already placed on myositis-specific autoantibodies as clinical decision-making tools.
\end{abstract}

Keywords: Myositis, Autoantibody

\footnotetext{
* Correspondence: s.tansley@nhs.net

${ }^{1}$ University of Bath, Bath, UK

Full list of author information is available at the end of the article
}

(c) The Author(s). 2020 Open Access This article is licensed under a Creative Commons Attribution 4.0 International License, which permits use, sharing, adaptation, distribution and reproduction in any medium or format, as long as you give appropriate credit to the original author(s) and the source, provide a link to the Creative Commons licence, and indicate if changes were made. The images or other third party material in this article are included in the article's Creative Commons licence, unless indicated otherwise in a credit line to the material. If material is not included in the article's Creative Commons licence and your intended use is not permitted by statutory regulation or exceeds the permitted use, you will need to obtain permission directly from the copyright holder. To view a copy of this licence, visit http://creativecommons.org/licenses/by/4.0/ The Creative Commons Public Domain Dedication waiver (http://creativecommons.org/publicdomain/zero/1.0/) applies to the data made available in this article, unless otherwise stated in a credit line to the data. 
Nearly half a century has elapsed since the identification of the first myositis-specific autoantibody (MSA) [1]. A further sixteen MSAs have subsequently been described, and MSAs are now detectable in over $60 \%$ of people with myositis [2-4]. MSAs are generally mutually exclusive and identify individual homogeneous patient subgroups. They are important prognostic biomarkers and, as such, may have a role in the development of more personalized approaches to disease management. The real "boom" period for MSA discovery was between 1999 and 2009, when over 40\% of the MSAs described to date were reported [5]. Initially, these newer MSAs remained largely in the research domain as the laboratory techniques required to identify them were highly specialized, low-throughput, and expensive. The recent emergence of commercially available immunoassays to detect MSAs has increased access to these investigations, offering rapid MSA characterization, at low cost and without the need for specialist expertise. These assays were promptly adopted by clinicians and are now in widespread clinical use via commercial testing. While essential if MSAs are to be utilized in routine clinical practice, the benefits of broader access to these tools have been somewhat offset by concerns regarding the reliability of these immunoassays, specifically a low sensitivity for some key autoantibodies and a high false-positive rate in healthy controls, as highlighted by ourselves and others [6-10]. Validating new testing methods in any rare disease is a challenge, and this is further compounded by the rarity of some of the MSAs themselves. For example, certain anti-synthetase MSAs included in commercial immunoassays are found in just $0.3 \%$ of myositis patients [2].

The International Myositis Assessment and Clinical Studies (IMACS) group is a coalition of health care providers and researchers with an interest in myositis who seek to facilitate collaborative international myositis research. Recognizing both the potential benefits and pitfalls of MSA testing, IMACS established a Myositis Autoantibodies Scientific Interest Group. Given the absence of any guidance in this area, the group first sought to determine how MSA testing is being used in current clinical practice. A health professional survey was conducted to determine participants' experience with myositis autoantibody testing, including data on their usual testing methods and practices. Study data were collected and managed using the REDCap online tool (https://projectredcap.org), hosted at the University of Bath [11]. A copy of the survey can be reviewed as supplementary material.

The survey was sent to all 530 IMACS members on 30 August 2019, and complete responses were received from 111 participants by the deadline of 30 September 2019. One hundred respondents were based at 65 institutions, across six continents. Eleven participants did not identify their institution and chose to complete the survey anonymously. Forty-two percent of respondents were based in USA/Canada, 21\% in Europe, and $14 \%$ in Asia. Enzyme immunoassay/ELISA was reported to be the most widely used MSA detection technique worldwide and was used by $46 \%$ of respondents' local laboratories. This was followed by the line blot, used by $37 \%$ of respondents' laboratories. In Europe, the market was dominated by the Euroimmun line blot; $74 \%$ of European respondents' local laboratories used a line blot and $48 \%$ the Euroimmun line blot. In USA/Canada, there was a more even spread of techniques used, with enzyme immunoassay/ELISA (36\%), line blot (19\%), and immunoprecipitation/immunoblot (23\%) all popular testing methodologies. In Asia, 50\% of respondents' local laboratories utilized the line blot and 56\% enzyme immunoassay/ELISA. Despite published concerns regarding the sensitivity/specificity of the various immunoassays, only $41 \%$ of respondents received guidance from their laboratory on the interpretation of positive results and $81 \%$ of participants stated that their laboratory failed to highlight discordant results obtained using multiple techniques (e.g., absence of cytoplasmic speckled staining on indirect immunofluorescence in association with a positive anti-synthetase antibody). This is a potential concern, as, with the more widespread availability of MSA detection methods, these tests are likely to be requested by non-expert users, who may lack the specialist knowledge required to interpret discordant or inconsistent results.

We found that $64 \%$ of the participants stated that they were confident with the results provided by their MSA testing laboratory. However, the same number of respondents also admitted that their confidence varied depending on the MSA in question, and this was largely due to concerns about false-positive and false-negative results. Despite these reservations, the majority of participants reported that MSA testing influenced their diagnostic confidence (83\%), the information they relayed to their patients on prognosis (86\%), further investigations planned $(81 \%)$, and even their recommended treatment (73\%). The latter finding was particularly surprising, given that the evidence base for pharmacological therapies in myositis is extremely limited, with support for the differential treatment response based on MSA positivity relying predominantly on the sub-analysis of a single randomized controlled trial $[12,13]$. Key survey findings are summarized in Fig. 1.

Our survey had several limitations, including a risk of bias and inaccuracy in data reporting (due to the possibility of participants completing the survey more than once and multiple participants from the same institution). Furthermore, the complex arrangements for 


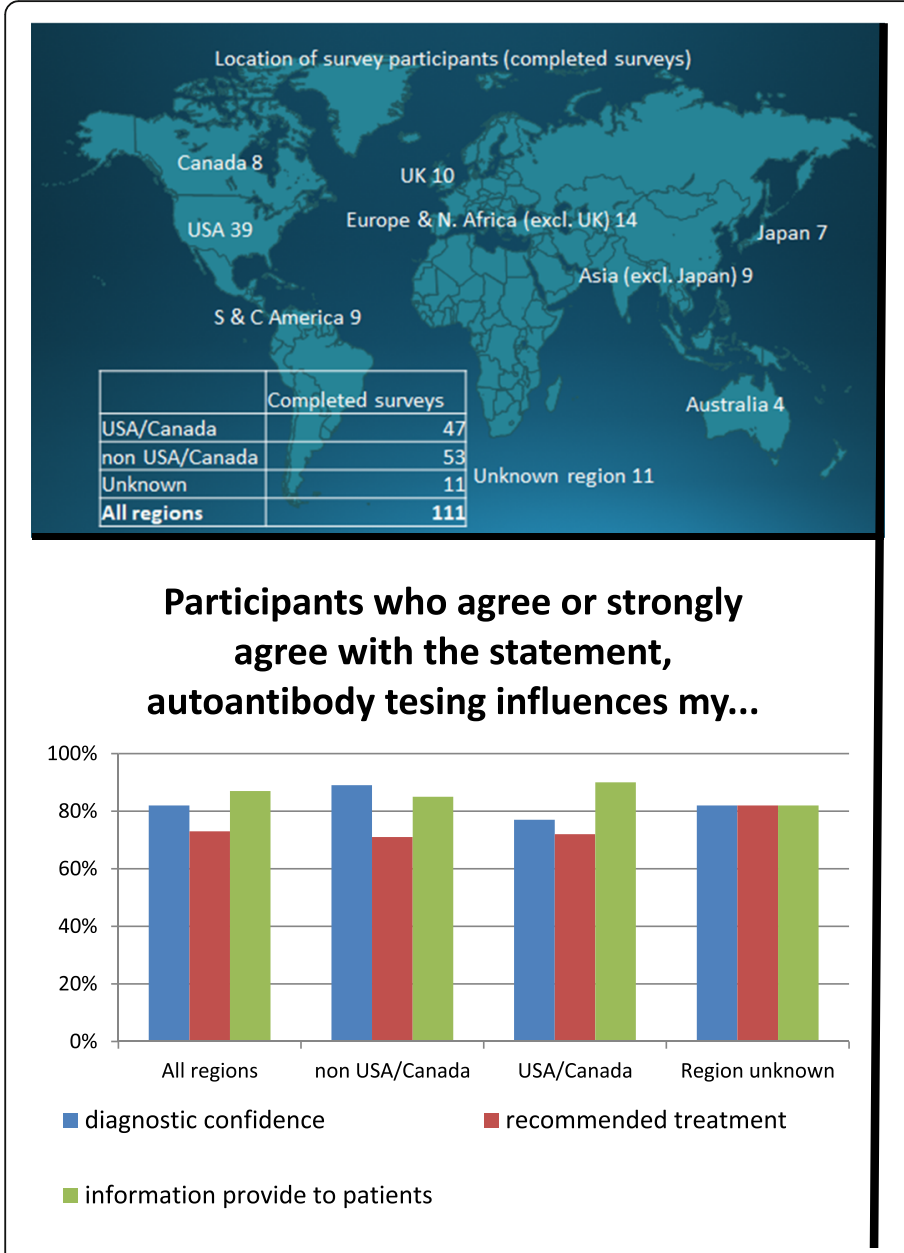

\section{Which type of assay does your local laboratory use for identifying myositis relevant autoantibodies?}

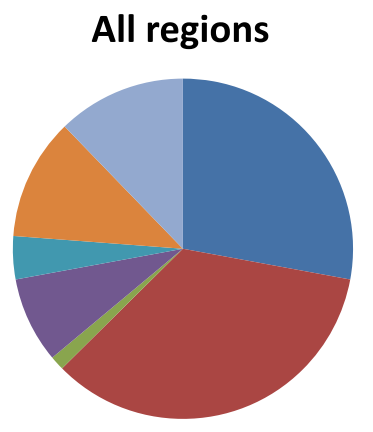

Line blot

Non USA/Canada

- Enzyme immunoassay/ELISA

- Laser bead

- Immunoprecipitation

- Other

Don't know

- Immunoprecipitation/immunoblot

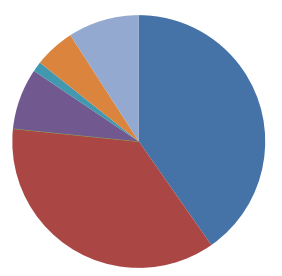

Region unknown

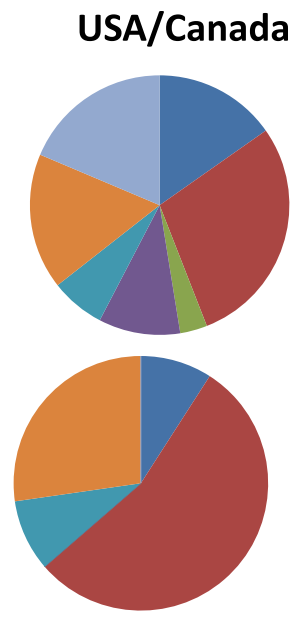

Fig. 1 A visual summary of key survey findings

autoantibody testing which exist at some institutions were not adequately captured by the survey. The survey predominantly captured the views of rheumatologists with an interest in myositis, who make up the majority of IMACS members (76\% of respondents were rheumatologists, $8 \%$ neurologists, $5 \%$ dermatologists, $11 \%$ other medical specialties and anonymous respondents). These views may therefore not be shared by other medical professionals.

Our results clearly demonstrate that MSA testing by commercial immunoassays is being used globally to inform clinical decision-making. Although our survey respondents all have expertise in myositis, more than $90 \%$ felt that more education was needed on the interpretation of autoantibody results and this should be urgently addressed. We have identified the key MSA testing methods used globally (enzyme immunoassay/ELISA and line blot). While there may be some variation between manufacturers, we know that certain assay types perform poorly for particular myositis specificities, for example, in a significant proportion of patients, anti-
TIF1y autoantibodies target a conformational epitope and therefore will not be detected by blotting-based assays [9]. Our findings will allow a better targeting of resources and further research on test result accuracy and comparability, in addition to educational resources, which should focus the most commonly used assay types. Our data also provide some justification for comparing novel testing methods to other commercial assays which could be considered "standard clinical practice" although we would argue that immunoprecipitation as a gold standard method would be preferable where available.

Broader access to MSA testing is welcomed for its potential to improve patient outcomes. However, assessing the reliability of current testing methods is essential given the importance placed on MSA as clinical decision-making tools. An understanding of the limitations of the chosen testing method and guidance on when and in whom MSA tests should be performed is vital. This data justifies ongoing work to answer these important questions. 


\section{Supplementary information}

Supplementary information accompanies this paper at https://doi.org/10. 1186/s13075-020-02210-2.

\section{Additional file 1.}

\section{Acknowledgements}

We are grateful to all those who responded to the survey including Brittany Adler, Rohit Aggarwal, Amita Aggarwal, Jakkrit Amornvit, Christine Anastasiou, Lilia Andrade-Ortega, Luis Arboleya, Dana Ascherman, Umesh Badrising, Richard Barohn, Murray Baron, Josiane Bourre-Tessier, Daniel Brito de Araujo, Hermine Brunner, Richard Campbell, Hector Chinoy, Leslie Crofford, Megan Curran, Katalin Dankó, Louise Pyndt Diederichsen, Mazen Dimachkie, Lyubomir Dourmishev, Jeffrey Dvergsten, Adriana Elias, Michael Feely, David Fernandez, Manabu Fujimoto, Abraham Garcia Kutzbach, Ignacio Garcia-De La Torre, Michael George, Takahisa Gono, Patrick Gordon, Latika Gupta, Raouf Hajji, Claas Hinze, Sabrina Hoa, Kristin Houghton, Christine Hsieh, Adam Huber, Marie Hudson, Maria Ibarra, Estíbaliz Iglesias, Cheilonda Johnson, Yasuhiro Katsumata, Raju Khubchandani, Hanna Kim, Drew Kurtzman, Océane Landon-Cardinal, James Lilleker, Daniel Lovell, Liangjing Lu, Ingrid Lundberg, Andrew Mammen, Liza McCann, Christopher Mecoli, Yoshinao Muro, Taio Naniwa, Merrilee Needham, Antonella Notarnicola, Chester Oddis, Amir Orandi, Charalampia Papadopoulou, Matthew Parker, Clarissa Pilkington, Pallavi Pimpale Chavan, Liza Rajasekhar, Ann Reed, Lisseth Rodas, Kelly Rouster-Stevens, Seward Rutkove, Lesley Ann Saketkoo, Adam Schiffenbauer, Elena Schiopu, Leslie Schrieber, Albert Selva-O'Callaghan, JeanLuc Senécal, Avinash Sharma, Susan Shenoi, David Sherry, Samuel Shinjo, Ho So, Charles Spencer, Brittany Stevenson, Cory Stingl, Grant Syverson, Piotr Szczesny, Eleni Tiniakou, Jiram Torres-Ruiz, Jiř́ Vencovský, Christian von Frenckell, Carlos von Mühlen, Victoria Werth, Erin Wilfong, Steven Ytterberg, Erick Adrian, and Zamora Tehozol.

\section{Authors' contributions}

NM supervised the project. ST drafted the survey in consultation with JP, JR, MK, and NM. JS created the REDCap survey, extracted the responses, and analyzed the data. LR distributed the survey via IMACS. AL and ST summarized the key findings. ST prepared the manuscript in consultation with JS, JP, AL, MK, LR, and NM. All authors and the IMACS scientific committee read and approved the final manuscript.

\section{Funding}

This project received no specific funding. This research was supported in part by the Intramural Research Program of the $\mathrm{NIH}$, National Institute of Environmental Health Sciences, and funding from the Bath Institute of Rheumatic Diseases.

\section{Availability of data and materials}

The dataset created and analyzed during the current study is available from the corresponding author on reasonable request.

\section{Ethics approval and consent to participate}

The study protocol received prior approval from the University of Bath research ethics service (REACH EP 18/19 040) as well as the IMACS Scientific Committee, and all respondents provided informed consent.

\section{Consent for publication}

Not applicable

\section{Competing interests}

M. Kuwana holds a patent on an anti-MDA5 antibody measuring system. JP has undertaken consultancy work for Actelion pharmaceuticals, Boehringer Ingelheim, and Sojournix Pharma outside of the submitted work. The remaining authors declare that they have no competing interests.

\section{Author details}

${ }^{1}$ University of Bath, Bath, UK. ${ }^{2}$ Keio University, Tokyo, Japan. ${ }^{3}$ National Institute of Health, Bethesda, MD, USA. ${ }^{4}$ Uppsala Universitet, Uppsala, Sweden.
Received: 2 March 2020 Accepted: 1 May 2020

Published online: 15 May 2020

\section{References}

1. Reichlin M, Fau - Mattioli M, Mattioli M. Description of a serological reaction characteristic of polymyositis. Clin Immunol Immunopathol. 1976;5(1):12-20.

2. Betteridge Z, Tansley S, Shaddick G, Chinoy H, Cooper RG, New RP, et al. Frequency, mutual exclusivity and clinical associations of myositis autoantibodies in a combined European cohort of idiopathic inflammatory myopathy patients. J Autoimmun. 2019;101:48-55.

3. Pluk H, van Hoeve Bj Fau - van Dooren SHJ, van Dooren Sh Fau - StammenVogelzangs J, Stammen-Vogelzangs J Fau - van der Heijden A, van der Heijden A Fau - Schelhaas HJ, Schelhaas Hj Fau - Verbeek MM, et al. Autoantibodies to cytosolic $5^{\prime}$-nucleotidase $1 \mathrm{~A}$ in inclusion body myositis. Ann Neurol 2013:73(3):397-407.

4. Betteridge Z, Chinoy H, Vencovsky J, Winer J, Putchakayala K, Ho P, et al. Identification of a novel autoantigen eukaryotic initiation factor 3 associated with polymyositis. Rheumatology. 2020;59(5):1026-30.

5. McHugh NJ, Tansley SL. Autoantibodies in myositis. Nat Rev Rheumatol. 2018;14(5):290-302

6. Espinosa-Ortega F, Holmqvist M, Alexanderson $H$, Storfors $H$, Mimori T, Lundberg IE, et al. Comparison of autoantibody specificities tested by a line blot assay and immunoprecipitation-based algorithm in patients with idiopathic inflammatory myopathies. Ann Rheum Dis. 2019;78(6):858-60.

7. Bundell C, Rojana-Udomsart A, Mastaglia F, Hollingsworth P, McLean-Tooke A. Diagnostic performance of a commercial immunoblot assay for myositis antibody testing. Pathology. 2016;48(4):363-6.

8. Mecoli CA, Albayda J, Tiniakou E, Paik JJ, Zahid U, Danoff SK, et al. Myositis autoantibodies: a comparison of results from the Oklahoma Medical Research Foundation myositis panel to the Euroimmun research line blot. Arthritis Rheumatol. 2020;72(1):192-4.

9. Tansley SL, Li D, Betteridge ZE, McHugh NJ. The reliability of immunoassays to detect autoantibodies in patients with myositis is dependent on autoantibody specificity, Rheumatology. keaa021, https://doi.org/10.1093/ rheumatology/keaa021.

10. Mahler M, Betteridge Z, Bentow C, Richards M, Seaman A, Chinoy H, et al. Comparison of three immunoassays for the detection of myositis specific antibodies. Front Immunol. 2019;10:848.

11. Harris PA, Taylor R, Thielke R, Payne J, Gonzalez N, Conde JG. Research electronic data capture (REDCap)--a metadata-driven methodology and workflow process for providing translational research informatics support. J Biomed Inform. 2009:42(2):377-81.

12. Vermaak E, Tansley SL, McHugh NJ. The evidence for immunotherapy in dermatomyositis and polymyositis: a systematic review. Clin Rheumatol. 2015;34(12):2089-95.

13. Aggarwal R, Bandos A, Reed AM, Ascherman DP, Barohn RJ, Feldman BM, et al. Predictors of clinical improvement in rituximab-treated refractory adult and juvenile dermatomyositis and adult polymyositis. Arthritis Rheumatol. 2014;66(3):740-9.

\section{Publisher's Note}

Springer Nature remains neutral with regard to jurisdictional claims in published maps and institutional affiliations. 